\title{
Taking a closer look at the pancreas
}

\section{K. Coppieters • M. von Herrath}

Published online: 24 October 2008

(C) Springer-Verlag 2008

Keywords Beta cell mass · Diabetes · Imaging · Intravital · Invasive $\cdot$ Non-invasive $\cdot$ Optical $\cdot$ Tomography

\begin{abstract}
Abbreviations
MRI magnetic resonance imaging

OPT optical projection tomography

PET positron emission tomography

Tregs regulatory $\mathrm{T}$ cells
\end{abstract}

Our current understanding of how pancreatic beta cell mass fluctuates during the course of diabetes development is largely based on indirect evidence. Various approaches based on in vivo metabolic testing have been described, each reflecting specific aspects of beta cell function [1]. These measurements are correlated with actual beta cell mass only to a certain extent, and were developed to allow estimates of mass following surgery, therapy or transplantation. Direct visualisation of beta cell mass by means of novel imaging technologies is anticipated to provide more detailed insight into the true kinetics of key processes such as physiological beta cell turnover, islet graft loss and the pathophysiology of type 1 and type 2 diabetes. Likewise, a substantial part of what we know about the autoimmune component of type 1 diabetes has historically been derived from ex vivo, cross-sectional studies in rodents. Improved intravital microscopy techniques could also shed new light on the in situ behaviour of immune cells-after all, seeing is believing.

In this issue of Diabetologia, Holmberg and Ahlgren review recent progress in the area of optical imaging technologies that is specifically applicable to monitoring the pancreas [2]. Although most investigators may be familiar with conventional non-optical imaging modalities such as magnetic resonance imaging (MRI) and positron

K. Coppieters $\cdot$ M. von Herrath $(\triangle)$

The La Jolla Institute for Allergy and Immunology,

9420 Athena Circle,

La Jolla, CA 92037, USA

e-mail: matthias@liai.org emission tomography (PET), and the ongoing endeavour to optimise these technologies for non-invasive monitoring of beta cell mass in patients, there are a number of lesser known optical strategies that are likely to contribute essential information in the near future. In an effort to improve our understanding of emerging technologies such as optical projection tomography (OPT), bioluminescence imaging, confocal microscopy and optical coherence tomography, the authors outline the characteristics, benefits and limitations of each technique. Perhaps one of the most elegant examples of this new generation of imaging modalities is optical projection tomography, an approach that has recently been advanced by the authors to allow for detailed whole organ assessment and accurate quantification of beta cell mass ex vivo. The three-dimensional OPT image provided in their current review showcases the remarkable quantitative resolution that is characteristic of OPT, enabling us to observe individual islets and their position relative to each other. The authors conclude that, although OPT and the other technologies discussed are at present merely suitable for pre-clinical research, they could prove clinically relevant in the long run.

So which questions need to be addressed by optical imaging? (see text box: Questions to be addressed by optical imaging). We and others have recently proposed a cyclical model for type 1 diabetes, describing the disease as a relapsing-remitting condition, based on our understanding of its key cellular constituents [3]. According to this hypothesis, the kinetics of beta cell degeneration are directed by the degree of epitope spreading, the rate of beta cell proliferation in response to autoimmune attack and the immune modulatory action of regulatory $\mathrm{T}$ cells (Tregs). As a result of the dominance of the autoreactive effector $T$ cell responses, beta cell mass will gradually decrease in atrisk individuals, ultimately leading to disease manifestation once a critical threshold has been passed. Support for the existence of this delicate balance was obtained in the NOD mouse, still the most widely studied animal model of type 1 diabetes. Sensitive longitudinal detection of alterations in beta cell mass during diabetes development in these animals may put this theory to the test and may 
unequivocally show whether or not beta cell loss occurs in a non-linear fashion.

\section{Questions to be addressed by optical imaging}

- Kinetics of beta cell loss during experimental diabetes

- Fate of islet transplants and how to prolong survival

- Overall effect of beta cell proliferation

- Dynamics and interaction pattern of Tregs

- Behaviour and interactions of autoreactive T cells

From a clinical perspective, significant efforts have been undertaken in search of a sensitive, safe and non-invasive method of monitoring islet grafts upon transplantation to assess early islet graft survival and to adjust immunosuppressive therapy if required. While investigators are mainly focusing on MRI and PET as the methodologies of choice to dissect the early fate of islet transplants, OPT, possibly in conjunction with two-photon laser scanning microscopy, could be of value for the study of the deteriorative process and the effect of experimental immunosuppressive regimens in animal models.

One of the most intriguing phenomena, as observed in NOD animals, is the early onset of a powerful beta cell proliferative response upon autoimmune infiltration of the islets [4]. With the beta cells going into rescue mode, it seems plausible that this will be reflected in terms of beta cell mass. A major proportion of current diabetes research is focused on the specific therapeutic facilitation of endogenous beta cell regeneration, and reproducible methods of studying the effects of such drugs on beta cell mass over time would be of high interest. Optical imaging could substitute for the rather inaccurate estimation by morphometric quantification to unveil more subtle changes following experimental therapy. Importantly, efficient imaging of beta cell mass during experimental treatment may expose disadvantages associated with anti-inflammatory drugs. The finding that certain common non-steroidal immunosuppressants negatively influence beta cell proliferation underscores the importance of monitoring this variable [5].

Regulatory $\mathrm{T}$ cells constitute another promising subject for therapeutic intervention and are principal players at the interface between autoreactive $\mathrm{T}$ cells and their beta cell targets. With the advent of two-photon laser scanning microscopy, as discussed by Holmberg and Ahlgren, it is now possible to visualise cellular interactions in real time. Tang and coworkers elegantly used this technology to assess the biological basis of regulatory $\mathrm{T}$ cell suppression in pancreatic draining lymph nodes [6]. Excitingly, they observed that both effector and regulatory $\mathrm{T}$ cells stably interact with islet antigen-bearing dendritic cells in the pancreatic lymph node, rather than with each other. However, there are many questions outstanding regarding the in situ behaviour of Tregs. For example, how do they affect autoaggressive $\mathrm{T}$ cells locally in the islets? To that end, we have recently proposed a surgical technique that will allow in vivo two-photon microscopy monitoring in the pancreas [7].

High-resolution optical technology also enables the visualisation of the actual culprits of type 1 diabetes, the autoreactive $\mathrm{T}$ cells. One seemingly simple, albeit unanswered, question is: how many beta cells are killed by one individual $\mathrm{T}$ cell entering the islets? How long do T cells remain in an islet upon initial entry and do they traffic between islets? Apart from monitoring the fate of islet transplants themselves in terms of beta cell mass, it would be interesting to see how autoreactive $\mathrm{T}$ cells interact with the transplanted beta cells in situ and how tolerance induction affects their infiltration and motility. These are fundamental problems for which, to date, no direct in vivo evidence is available.

Finally, it is evident that any set of data generated by new imaging techniques, each associated with inherent limitations, must be correctly analysed to avoid misinterpretation. With regard to the analysis of beta cell mass, correlation with the existing metabolic tests is mandatory. It may well be that the outcome of such comparison will be surprising. Indeed, even though C-peptide is now widely regarded as the preferred outcome measure during clinical trials, its precise relation to beta cell mass remains questionable as it rather reflects the functionality of the available beta cell pool [8]. In this respect, one should take great care to distinguish between functional beta cell mass and metabolically inactive beta cells, as reports in the NOD mouse have clearly shown that there is a discrepancy between the two [4]. A particular requirement for the use of two-photon imaging is that significant numbers of events need to be monitored to yield conclusive data on variables such as $\mathrm{T}$ cell velocity and migration pattern. Provided these considerations are taken into account, the optical imaging technologies reviewed by Holmberg and Ahlgren represent promising tools to advance our understanding of the pathophysiology of type 1 diabetes and may lead to the design of more accurate non-invasive imaging modalities in humans.

Acknowledgements $\mathrm{K}$. Coppieters is supported by the D. Collen Research Foundation, the Belgian American Educational Foundation (BAEF) and the Commission for Educational Exchange between the United States of America, Belgium and Luxembourg (The Fulbright Program).

Duality of interest The authors declare that there is no duality of interest associated with this manuscript. 


\section{References}

1. Robertson RP (2007) Estimation of beta-cell mass by metabolic tests: necessary, but how sufficient? Diabetes 56(10): 2420-2424

2. Holmberg DA, Ahlgren U (2008) Imaging the pancreas: from ex vivo to non-invasive technology. Diabetologia doi:10.1007/s00125008-1140-7

3. von Herrath M, Sanda S, Herold K (2007) Type 1 diabetes as a relapsing-remitting disease? Nat Rev 7:988-994

4. Sherry NA, Kushner JA, Glandt M, Kitamura T, Brillantes AM, Herold KC (2006) Effects of autoimmunity and immune therapy on beta-cell turnover in type 1 diabetes. Diabetes 55:3238 3245

5. Nir T, Melton DA, Dor Y (2007) Recovery from diabetes in mice by beta cell regeneration. J Clinical Invest 117:2553-2561

6. Tang Q, Adams JY, Tooley AJ et al (2006) Visualizing regulatory $\mathrm{T}$ cell control of autoimmune responses in nonobese diabetic mice. Nat Immunol 7:83-92

7. Martinic MM, von Herrath MG (2008) Real-time imaging of the pancreas during development of diabetes. Immunol Rev 221:200-213

8. Palmer JP, Fleming GA, Greenbaum CJ et al (2004) C-peptide is the appropriate outcome measure for type 1 diabetes clinical trials to preserve beta-cell function: report of an ADA workshop, 21-22 October 2001. Diabetes 53:250-264 